\author{
Military Technical \\ College \\ Kobry Elkobbah, \\ Cairo; Egypt
}

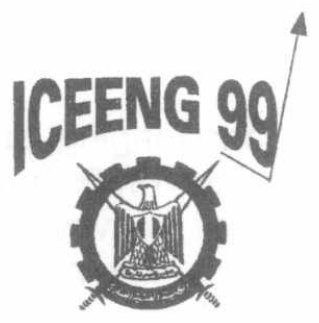

\author{
$2^{\text {nd }}$ International \\ Conference on Electrical \\ Engineering \\ ICEENG 99
}

\title{
A Study and Implementation of Optical Laser-Based Ranging Systems
}

\author{
G. Fahim*, A.S. GERGES**, E.A.Mehanna**, and S.M.Alian ${ }^{* *}$
}

\section{ABSTRACT:}

The increased requirements on ranging precision in many military, industrial and astronomical applications illustrate the importance of the Laser ranging systems (called Laser radar) as a modern ranging system. In this paper, the authors study the main features of laser ranging systems in its two main modes, the pulsed mode and the continuous wave subcarrier mode (CWSM). The main advantages of each mode are discussed then proved out by experimental data given by the satellite ranging system Helwan and an experimental setup built on the bases of CWSM. These results show good agreement with that expected theoretically.

\section{KEY WORDS:}

Laser ranging, Laser rader, Laser range finder, Lidar, Ladar, Laser metrology, Laser systems, Optical ranging.

\footnotetext{
* National Research Institute of Astronomy and Geophysics, Cairo, Egypt,

** Egyptian Armed Forces.
} 
Proceedings of the $2^{\text {nd }}$ ICEENG Conference, 23-25 Nov. 1999

\begin{tabular}{|l|l|}
\hline $\mathrm{PH}-1$ & 2 \\
\hline
\end{tabular}

\section{INTRODUCTION:}

Lidar [1-3], light detection and ranging, or ladar, laser detection and ranging systems were first introduced in the late 1960s and early 1970 s. Their initial use was applied to many applications such as surveying, tactical laser range finders and beam rider missile guidance systems. The main advantages of ladars are its small size and very high angular accuracy values.

In this work the principles of laser ranging measurements is described in its two main modes the pulsed mode and the continuous wave subcarrier mode (CWSM). The main advantages of each mode are discussed. Two systems are employed to illustrate the main advantages of each system; the first is the pulsed laser ranging system (Helwan) used to collect a new set of data for the satellite " Lageos -1 ". The second is a high accuracy CWSM based system designed and implemented as a small range laser ranging system with very small blind range.

\section{THE LADAR RANGE EQUATION:}

Optical radar methods for distance measurement are essentially based upon the determination of the transit time of flight between a fiducial point and the target of interest. There are basically two approaches to this measurement $[4,5]$ :

1. Direct - Pulsed mode: where the round trip time (time of flight $\tau_{d}$ ) of a short pulse of light is directly timed. The range $R$ is given directly by the equation:

$$
\mathrm{R}=\mathrm{c} \tau_{\mathrm{d}} / 2
$$

Where $\mathrm{C}$ is the free space speed of light. This technique is mainly used for measurement of medium and long distances, as example for satellite ranging systems. Due to the very fast electronics required for this approach, few attempts have been made to exploit this technique for short-range distance measurement

2. Indirect -Continuous wave subcarrier modulation (CWSM) mode: where the intensity of the source is modulated at high frequency and the distance is determined from the change in phase induced in the subcarrier during the transit of the light.

\section{Pulsed mode laser ranging:}

In this technique, the laser transmitter transmits a very short, high power optical pulse using Q-switching [6], or any other technique of producing very short optical pulses. By measuring the time delay of the reflected pulse, the range can be determined directly from equation (1).

The maximum unambiguous range $R_{\max }$ is a function of the inter-pulse period $T$, as given by $\left(R_{\max }=c T / 2\right)$. Beyond this range, the elapsed time between transmitted and received pulses will include multiples of $T$, making the range ambiguous. Practically, $T$ is very large such that we can say that $R_{\max }$ is unlimited. Another factors that may limit the maximum range $R_{\max }$ are the optical beam geometry, atmospheric attenuation, target cross-section,...., etc [7]. The pulse width $\tau_{p}$ is chosen to be very small since the minimum detectable range (dead range) $R_{\text {min }}$ should be smaller or equal to $\left(c \tau_{p} / 2\right)$ to avoid the overlapping of the transmitted and received pulses. It is well known that the optical energy associated with the pulse is $E_{0}=P \tau_{p}$ where $P$ is the optical power during the pulse. As $\tau_{p}$ decreases, the optical energy decreases, which requires a huge power to give enough energy. The main advantages of this technique, is the unlimited range (from signal processing point of view). But the main drawback of pulsed technique is that there will be a blind range (about $145-200 \mathrm{~m}$ ) through which no target can be detected. This blind range 
comes from the very short time of flight of light that overlaps the transmitted and received pulses, hence the time of flight can not be measured.

Continuous wave subcarrier modulation (CWSM)

CWSM is one of the standard techniques used in both conventional and optical radars. It is utilized in optical radar when the displacement resolution required is much greater than the wavelength of the source. The basis of this method is that the wavelength of the source can be increased by several orders of magnitude to an effective wavelength $\lambda_{m}$ by imposing an intensity modulation of the form:

$$
\mathrm{I}=\mathrm{I}_{0} \sin \omega_{\mathrm{m}} \mathrm{t}
$$

where $I_{0}$ is the amplitude and $\omega_{m}$ is the angular frequency. Consider a diode laser driven by sinusoidal input current that produces intensity modulated laser beam, hence the laser beam intensity will be in the form:

$$
\mathrm{I}_{\mathrm{T}}=\mathrm{I}_{\mathrm{OT}} \sin \left(\omega_{\mathrm{m}} \mathrm{t}+\phi_{\mathrm{L}}\right)
$$

where $I_{T}$ is the intensity of the transmitted wave, $I_{O T}$ is the maximum transmitted intensity, and $\phi_{\mathrm{L}}$ is the phase shift in the laser. After the laser beam has been collimated, it is splitted by a beam splitter into two beams. The first beam is intercepted by a photodetector, where it is taken as a reference signal. The second beam travels to the target, where it is reflected back and focused by converging lens on a photodetector. The output signals from the two photodetectors are amplified and reshaped. The reference signal will be in the form:

$$
I_{R 1}=I_{o R 1} \sin \left(\omega_{m} \hat{\imath}+\phi_{L}+\phi_{d}+\phi_{a}\right)
$$

where $I_{R 1}$ is the intensity of the reference signal, $I_{O R}$ is the maximum intensity of the reference signal, and $\phi_{d}$ and $\phi_{a}$ are the phase increase due to the detection and amplification of the light at the receiver. The target signal will be in the form:

$$
I_{R 2}=I_{o R 2} \sin \left(\omega_{m} t+\phi_{L}+\phi_{R}+\phi_{d}+\phi_{a}\right)
$$

where $I_{R 2}$ is the signal intensity reflected from the target signal, $I_{O R 2}$ is its maximum intensity and $\phi_{R}$ is the phase shift introduced due to flight of light a distance $2 R$, where $R$ is the range to be determined and is given by:

$$
\phi_{\mathrm{R}}=\mathrm{k}_{\mathrm{m}} \cdot 2 \mathrm{R}
$$

where $k_{m}=2 \pi / \lambda_{m}$ is the angular wave number, and $\lambda_{m}$ is the modulation wavelength. The two signals (reference signal and signal from the target) are amplified then reshaped into pulses and compared in a phase detector where the phase difference $\Delta \phi$ between these two signals is measured, where $\Delta \phi$ is given by:

$$
\Delta \phi=\phi_{\mathrm{R}}
$$

The phase difference $\Delta \phi$ is directly proportional to the range and given by:

$$
R=c(\Delta \Phi) / 4 \pi f_{m}
$$

Where $f_{m}=c / \lambda_{m}$ is the modulation frequency. Thus the range $R$ can be measured.

The accuracy of the range measurement depends on the accuracy with which the phase difference $\Delta \phi$ can be measured $[8,9]$. The maximum range $R_{\max }$ decreases as the modulating frequency $f_{m}$ increases. The choice of the modulating frequency doesn't only depend on the maximum range $R_{\max }$, but also on the required accuracy of measurement to be achieved[7]. It is important to define the main features of CWSM system, which are:

(i) The Maximum Unambiguous Range $\left(\boldsymbol{R}_{\max }\right)$ : The maximum value of phase shift $\left(\Delta \phi_{\max }\right)$ equals $(2 \pi)$ radian. This corresponds to the maximum unambiguous range of the system $R_{\max }$ that can be calculated from: 


$$
\mathrm{R}_{\max }=\frac{\mathrm{c}}{4 \pi \mathrm{f}_{\mathrm{m}}}(2 \pi)=\frac{\mathrm{c}}{2 \mathrm{f}_{\mathrm{m}}}=\frac{\lambda_{\mathrm{m}}}{2}
$$

\begin{tabular}{|l|l|}
\hline PH-1 & 4 \\
\hline
\end{tabular}

which shows that as $f_{m}$ decreases ( $\lambda_{m}$ increases) the value of maximum unambiguous range increases. Theoretically, the value of $R_{\max }$ is unlimited, but it is well known that the noise level of the system at low frequencies is very high which limits the minimum value of modulating frequency and hence the maximum value of $R_{\max }$.

(ii) The Minimum Detectable Range $\mathbf{R}_{\min }$ : The minimum detectable range $R_{\min }$ which is governed by the noise level of the system. If the noise level is $\mathrm{N}$, and the signal level is $\mathrm{S}$, the minimum detectable phase shift is given by:

$$
\pm \Delta \phi_{\min }= \pm \sin ^{-1}(\mathrm{~N} / \mathrm{S}) \text {, }
$$

Provided that $\mathrm{N} \leq \mathrm{S}$ which proves that as the signal to noise ratio increases, $\Delta \phi_{\min }$ decreases. If equation (8) and (10) are combined, then the minimum detectable range is given by:

$$
\mathrm{R}_{\min }=\frac{\mathrm{c} \Delta \phi_{\min }}{4 \pi \mathrm{f}_{\mathrm{m}}}
$$

For the same value of $\Delta \phi_{\min }$, the minimum detectable range $R_{\min }$ decreases as $f_{m}$ increases.

(iii) The system Dynamic Range: The system dynamic range of $R_{d}$ is defined as the ratio of the maximum unambiguous range to the minimum detectable range. In our system, the dynamic range is given by:

$$
\mathrm{R}_{\mathrm{d}}=\frac{\mathrm{R}_{\max }}{\mathrm{R}_{\min }}=\frac{2 \pi}{\Delta \phi_{\min }},
$$

which indicates that for the same value of $\Delta \phi_{\min }$, the dynamic range is independent of the modulating frequency $f_{m}$ and increases as the value of $\Delta \phi_{\min }$ decreases, which shows the great importance for the system enhancement to reduce the noise level in the system. Theoretically, $\Delta \phi_{\min }$ is the minimum detectable phase shift of the phase detector, but practically, its value depends on the used electronics. In our case, the value of $\Delta \phi_{\min }$ is $1 \mathrm{~m}$ radian, but practically its value becomes $4.188 \mathrm{~m}$ rad due to electronics, which results in the decrease of the dynamic range from 6283.185 to 1500.

\section{EXPERIMENTAL SYSTEMS:}

\section{a) Pulsed system:}

A block diagram of the satellite laser ranging system Helwan is shown in figure (1). A CCD camera is used for star tracking which gives a synchronization signal to the whole system when the star is locked. This signal triggers a Q-Switched Nd-YAG laser that produces very narrow (17 ps), very powerful ( $30 \mathrm{~mJ} / \mathrm{pulse}$ ) optical pulses of wavelength 0.53 microns. A trans-receiver telescope is used to send these pulses to the target then receives them back. A digital control and computing system is used to statistically determines the range of the satellite. At 19:13 during the night of $18 / 7 / 1999$ more than 230 laser pulses were fired towards the satellite "Lageos -1 ". The time of flight of each pulse was observed then the range residuals, the difference between the observed time and that calculated for the satellite (the O - C residuals), is determined. 


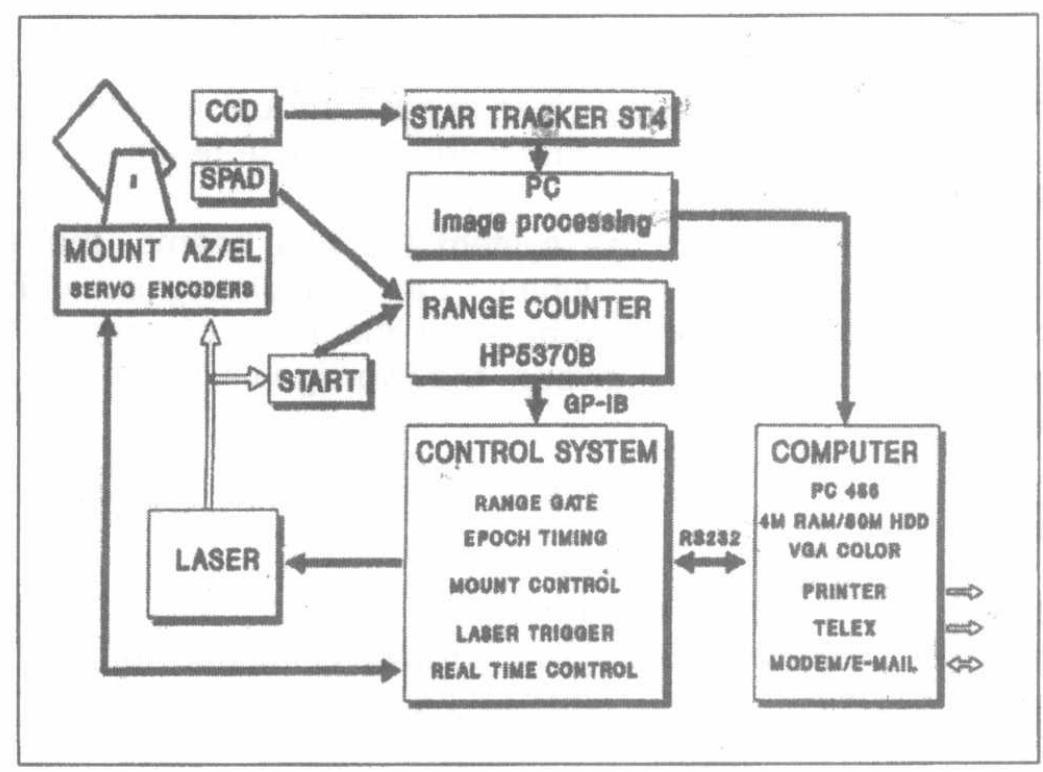

Fig.(1): A Simplified block diagram of the pulsed mode laser ranging station Helwan

\section{b) Realization of a CWSM laser ranging system:}

The system was designed to cover a range of $150 \mathrm{~m}$, with accuracy of $\pm 10 \mathrm{~cm}$ , and dynamic range of 1500. A schematic diagram of the system is shown in Fig.(2). It consists of an optical transmitter and optical receiver. The transmitter, which produces a collimated, intensity modulated laser beam, consists of a stable oscillator to modulate the diode laser in range of frequencies $0.5 \mathrm{MHz}$ to $3.5 \mathrm{MHz}$. A diode laser module RS 194-004 was used to produces a beam of wavelength $670 \mathrm{~nm}$ and output power of $3 \mathrm{~mW}$. An optical system is used to focus the beam onto the target.

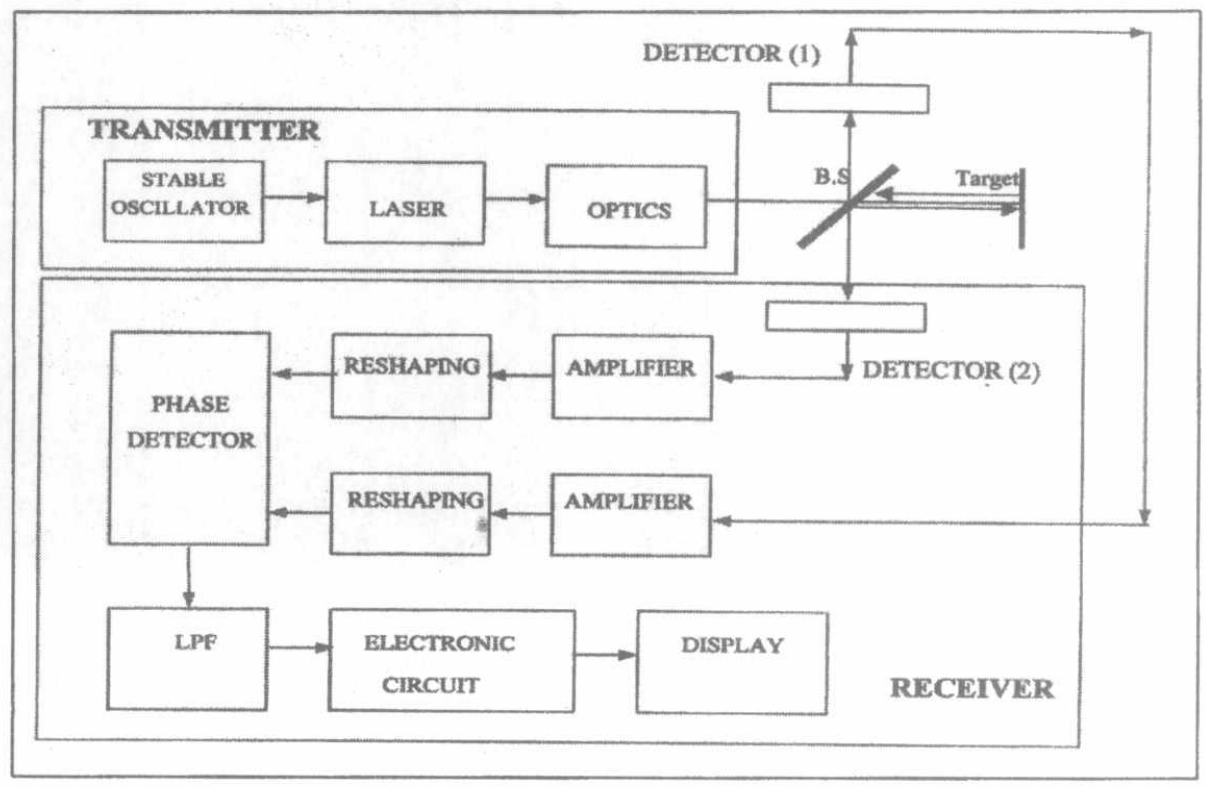

Fig. (2): Block diagram of the implemented CWSM laser ranging system 
The modulated laser beam is then pass through a beam splitter that divides the laser beam into two parts the first is the reference beam and the second is that transmitted to the target.

In the receiving part of the system the two beams, the reference and the reflected beams are detected, where the modulating sinusoidal waveform is recovered, then amplified and reshaped in the form of pulses. A phase detector detects the phase difference between the two signals, which are calibrated to give the range directly.

\section{RESULTS:}

a) Pulsed laser ranging system ( Helwan):

Figure (3) shows the histogram drawn, using polynomial data fitting, for the range residuals for the satellite Lageos- 1 which gives a mean value of $0.41413 \mathrm{~ns}$ ( or $12.4239 \mathrm{~cm}$ ), which was expected for the laser used.

b) CWSM laser ranging system:

A $1.00 \mathrm{MHZ}$ sinusoidal signal is used to modulate the laser diode. This frequency is chosen to give a reasonable signal to noise ratio and to satisfy our requirements for the maximum range to be covered $(150 \mathrm{~m})$. The target is placed at different ranges, and the corresponding values of phase shift $\Delta \phi$ are measured. These results are illustrated in Fig.(4). The results show a good linearity which may be represented by $R=\alpha(\Delta \phi) \pm \beta$, where the mean values of $\alpha$ and $\beta$ are $23.8732 \mathrm{~m} / \mathrm{rad}$ and $7.037 \mathrm{E}-08 \mathrm{~m}$ respectively.

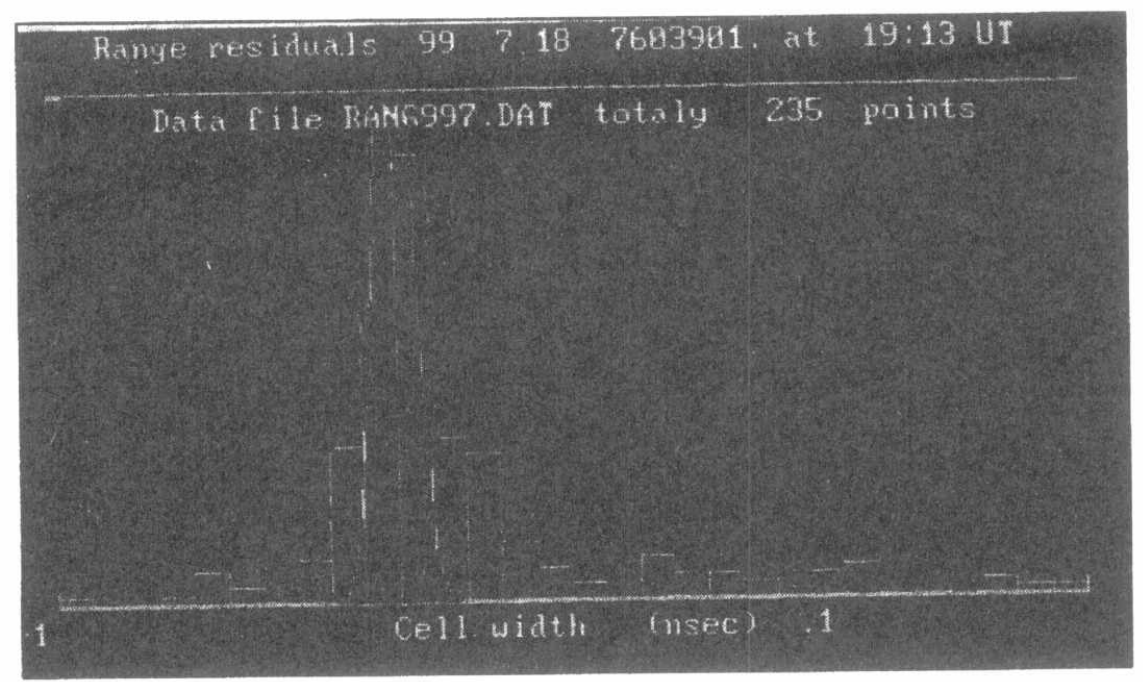

Fig.(3): Range residuals for Lageos-1 


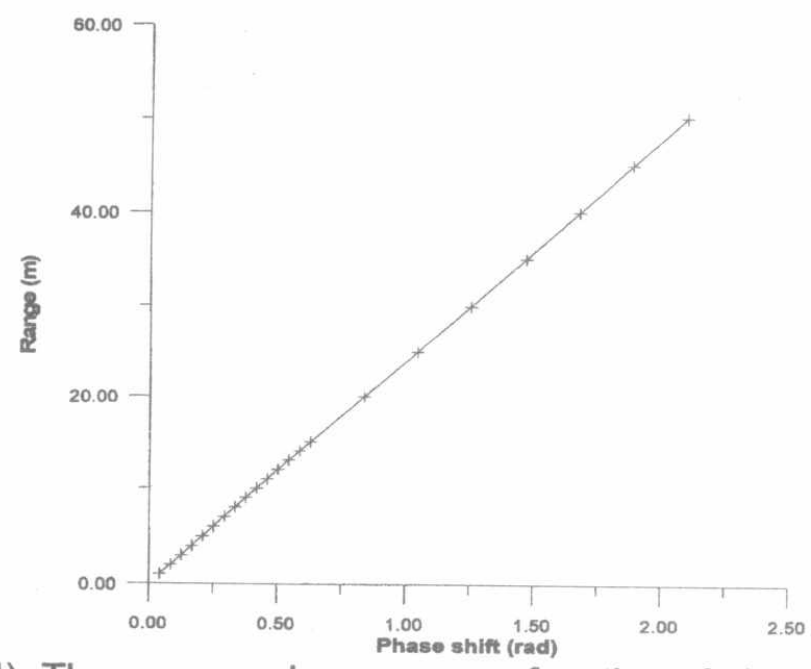

Fig. (4): The measured ranges as a function of phase shift.

For each position of the target, the resolution in range $\Delta R$ was measured, by moving the target by a micro-translation stage till the output of the phase detector shows a change in the value of $\Delta \phi$. In our system, the mean value of the signal to noise ratio $(\mathrm{S} / \mathrm{N})$ was 246.7 , which results in a mean value of phase resolution $\Delta \phi_{\min }$ of $\pm 4.188 \mathrm{~m}$ rad which corresponds to a range resolution of $96.7193 \mathrm{~mm}$.. The measured values of $\Delta R$ are shown in Figure (5).

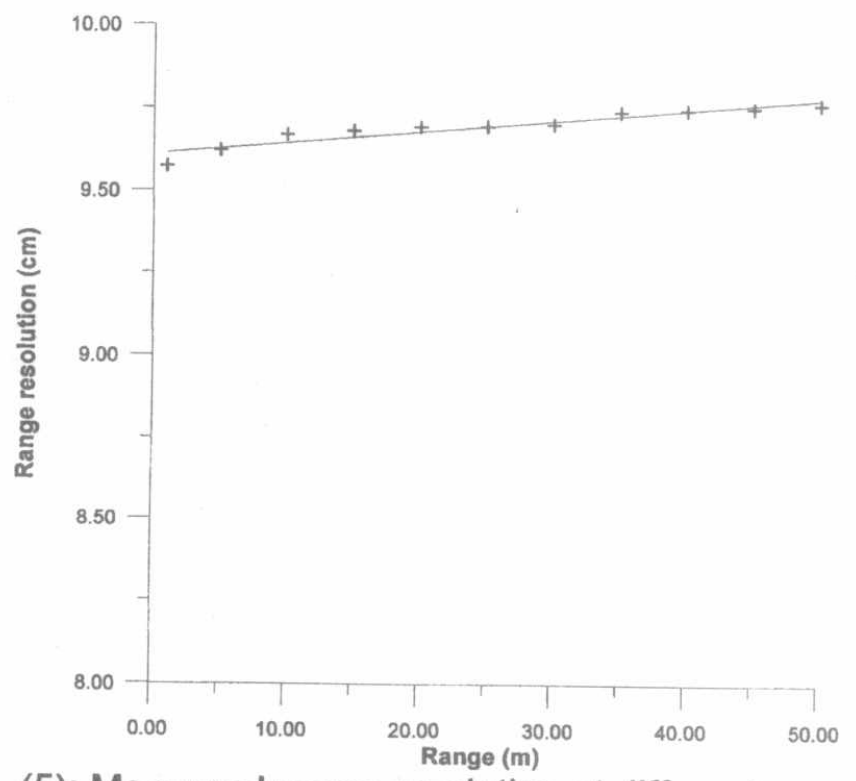

Fig. (5): Measured range resolution at different ranges.

The results show nearly constant resolution at small ranges, but as range $R$ increase, an increase in $\Delta R$ was measured. This increase in $\Delta R$ at higher values of $R$ is expected since the divergence of the laser beam will lead to a lower level of the reflected signal; and hence, less values of $S / N$ ratio and higher values of $\Delta \phi_{\min }$.

To test the dependence of $\Delta R$ on modulating frequency $f_{m}$, the target was fixed at $10 \mathrm{~m}$ range, while $\Delta R$ was measured as a function of $f_{m}$, which is adjusted to 
$0.5 \mathrm{MHz}, 1 \mathrm{MHz}, 1.5 \mathrm{MHz}, \ldots .$. , and $3.5 \mathrm{MHz}$. The results are shown in Figure (6), which show good agreement with the theoretical study.

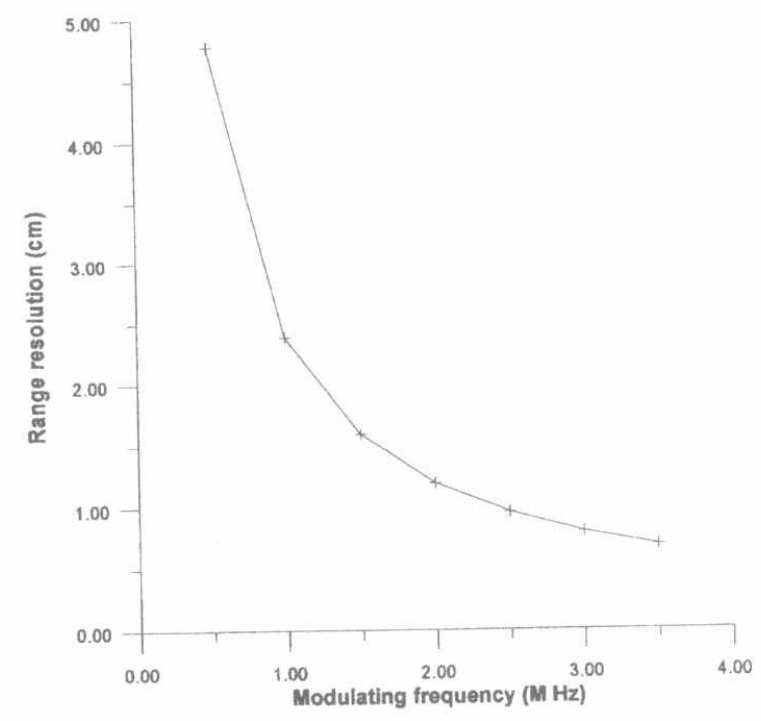

Fig. (6): Measured range resolution at different modulating frequencies.

\section{CONCLUSIONS:}

The main features of laser ranging systems in its two main modes, the pulsed mode and the continuous wave subcarrier mode (CWSM) have been studied. The main advantages of each mode have been discussed then proved out of experimental data. The following table summarizes the main features of each technique.

\begin{tabular}{|c|c|c|}
\hline Feature & \multicolumn{1}{|c|}{ Pulsed technique } & \multicolumn{1}{c|}{ CWSM technique } \\
\hline Max Range & $\begin{array}{l}\text { - Theoretically unlimited } \\
\text { - Practically limited by the } \\
\text { intensity of signal returning } \\
\text { back to receiver }\end{array}$ & $\begin{array}{l}\text { - Limited by a phase delay } \\
\text { of } 2 \pi \text { rad. }\end{array}$ \\
\hline Min Range & $\begin{array}{l}\text { Limited by the pulse } \\
\text { width ( blind range) }\end{array}$ & $\begin{array}{l}\text { - Theoretically unlimited. } \\
- \text { Practically limited by } \\
\text { phase which depends on } \\
\text { S/N ratio }\end{array}$ \\
\hline Use & $\begin{array}{l}\text { Very large and medium } \\
\text { ranges }\end{array}$ & \multicolumn{2}{c|}{ Short ranges } \\
\hline
\end{tabular}

Experimental results out of two laser ranging systems, the first is ready made while the second is designed and implemented in the lab, are given.

Based on laser pulsed range system the position of the satellite" Lageos-1" is measured with an accuracy of $12.4239 \mathrm{~cm}$. Based on continuous wave subcarrier modulation, a short range, laser range finder of maximum range $150 \mathrm{~m}$, was designed and implemented. The system was tested at different modulating frequencies and different ranges. It was proved that as the modulating frequency 
increases, the range resolution of the system becomes better. A signal to noise ratio of $23.92 \mathrm{~dB}$ was obtained, which resulted in a phase resolution of $\pm 4.188 \mathrm{~m}$ rad at modulating frequency of $1 \mathrm{MHz}$ that gave a range resolution of $96.7193 \mathrm{~mm}$.

\section{REFERENCES:}

[1] G.Fahim, Kh.I.Khalil and Y.S.Hanna," Data Analysis procedure of Helwan Satellite Laser Station", Bulletin of National Research Institute of Astronomy and Geophysics, Vol.XI, 1995, P.1.

[2] Hovanessian S. A., "Radar System Design and Analysis", Artech House, (1984).

[3] Barton, D. K., "Modern Radar System Analysis", Norwood MA, Artech House, (1988).

[4] Zmuda H. and Toughlian E. N., "Photonic Aspects of Modern Radar", Norwood MA, Artech House, (1992).

[5] James T. L. and David E. P., "Industrial Lasers and Their Applications", Prentice Hall Press, (1992).

[6] Grattan K.T.V. and Meggitt B. T., "Optical Fiber Sensor Technology", Cambridge University Press, (1995).

[7] Chin L., "Elements of optoelectronics and fiber optics", R.R. Donnelley \& Sons Company, (1996).

[8] M.M.Ahmed and A.S.Gerges, " Study and implementation of a laser range finder with very small blind range", Technology and Armament magazine, Vol.13-2, Jan.1998, P.91

[9] Webb D.J., Taylor R.M., Jones J.D.C., and Jackson D.A., Optics communications, No. 66, pp-245, (1988). 
